Check for updates

Cite this: Mater. Adv., 2022, 3,1556

Received 9th December 2021. Accepted 17th December 2021

DOI: 10.1039/d1ma01166a

rsc.li/materials-advances

\section{Preparation of mesh-like collagen scaffolds for tissue engineering}

\begin{abstract}
Yan Xie, (D) ab Naoki Kawazoe, ${ }^{a}$ Yingnan Yang ${ }^{c}$ and Guoping Chen (D) *ab
Collagen is an attractive biomaterial to construct scaffolds for tissue engineering and biomedical applications. Mesh-like collagen scaffolds were prepared using a synthetic poly(D,L-lactic-co-glycolic acid) (PLGA) mesh as a template. Collagen microsponges were introduced in the openings of a PLGA mesh to prepare a PLGA-collagen composite mesh, or a collagen coating layer was formed on a PLGA mesh to construct a collagen-coated PLGA mesh. Collagen mesh with large openings (collagen mesh-L) or small openings (collagen mesh-S) was prepared after selective removal of PLGA mesh from the PLGA-collagen composite mesh or collagen-coated PLGA mesh, respectively. The structure of collagen meshes was controlled by the porous structure of precursor meshes. Collagen mesh-S had a hierarchical structure composed of collagen microsponges in the openings of collagen mesh-L. The collagen meshes supported human skin fibroblast adhesion and promoted cell proliferation. In particular, collagen mesh-S showed the highest promotive effects on cell adhesion, homogenous distribution, cell proliferation and dermal tissue regeneration because of its small openings. Fibroblasts cultured in collagen mesh-S expressed various kinds of growth factors that could stimulate the proliferation of fibroblasts and the rapid formation of new dermal tissue. Homogenous dermal tissue was regenerated when fibroblasts were cultured in collagen mesh-S. The template method provides a useful method for mesh-like collagen scaffold preparation.
\end{abstract}

\section{Introduction}

Collagen-based biomaterials have been widely used to prepare porous scaffolds for tissue engineering because they are the most abundant structural proteins in vertebrates and play important roles in controlling cell functions such as cell adhesion, proliferation and differentiation. ${ }^{1-3}$ Collagen porous scaffolds have been applied for tissue engineering of cartilage, meniscus, bone, ligament, nerve, skin, and related structures. $^{4-6}$ Collagen can be used alone or in combination with other biomaterials, such as synthetic polymers, ceramics and other extracellular matrix components. ${ }^{7,8}$

There are many methods for the preparation of collagenbased scaffolds. ${ }^{9-11}$ Freeze dying is one of the frequently used methods. ${ }^{12}$ The freeze-drying process involves freezing an aqueous solution of collagen or collagen gel at a low temperature and a subsequent sublimation of the ice crystals by vacuum at low

\footnotetext{
${ }^{a}$ Research Center for Functional Materials, National Institute for Materials Science, 1-1 Namiki, Tsukuba, Ibaraki 305-0044, Japan.

E-mail: Guoping.CHEN@nims.go.jp; Fax: +81-29-860-4673; Tel: +81-29-860-4496

${ }^{b}$ Department of Materials Science and Engineering, Graduate School of Pure and

Applied Sciences, University of Tsukuba, 1-1-1 Tennodai, Tsukuba

Ibaraki 305-8577, Japan

${ }^{c}$ Graduate School of Life and Environmental Science, University of Tsukuba, 1-1-1

Tennodai, Tsukuba, Ibaraki 305-8572, Japan
}

temperature. The freezing temperature and freezing rate have some effects on the porous structure of collagen scaffolds. A unidirectionally structured collagen sponge was prepared by a unidirectional freezing-drying method. ${ }^{13,14}$ However, it has been difficult to precisely control the pore structure, especially the interconnectivity, of collagen-based scaffolds. The template method is a simple and effective approach to prepare ordered porous materials with precisely defined and controlled porous structures. $^{15,16}$ Templates such as ice crystals, colloidal crystals, salts and polymer microspheres have been used. ${ }^{17,18}$ Porous scaffolds used for tissue engineering generally require microscale pores with highly interconnected pore structures. Freeze-drying and porogen leaching methods sometimes exhibit poor interconnectivity unless proximate pore-forming template particles contact each other. Connection of the template particles before introduction of the base materials can improve the interconnectivity of porous scaffolds. Cubic close-packed lattices of monodispersed polymer microspheres have been used to increase the interconnectivity of porous scaffolds. ${ }^{15,19}$ These methods can be used for the preparation of thick collagen scaffolds. However, it is difficult to prepare thin mesh-like collagen scaffolds with good interconnectivity, although thin collagen scaffolds have broad applications for tissue engineering. ${ }^{20,21}$

In this study, we prepared mesh-type collagen scaffolds using a template method. A poly(DL-lactic-co-glycolic acid) 
(PLGA) knitted mesh was used as the template. Collagen meshes with large and small openings (pores) were prepared. First, a composite mesh of PLGA knitted mesh and collagen was prepared by forming web-like collagen microsponges in the openings of the PLGA mesh or by coating a collagen thin layer along the skeleton of the PLGA mesh. Then, the PLGA mesh was selectively dissolved by treatment with alkaline aqueous solution. Collagen meshes were formed after removal of the PLGA mesh template. Collagen meshes promoted the adhesion and proliferation of human dermal fibroblasts for dermal tissue regeneration.

\section{Experimental}

\section{Preparation of collagen meshes}

The preparation scheme is shown in Fig. 1. A mesh of synthetic polymer, poly(D,L-lactic-co-glycolic acid) (PLGA), was used as a template. A collagen coating layer was formed on the skeleton of the PLGA mesh, or collagen microsponges were formed in the interstices of the PLGA mesh. Then, the PLGA skeleton was selectively removed. Thin collagen meshes were prepared after removal of the PLGA template.

Two types of thin collagen mesh with small and large openings were prepared. Collagen mesh with small openings (collagen mesh-S) was prepared by selectively removing the PLGA template from a PLGA-collagen composite mesh. First, a PLGA-collagen composite mesh was prepared by forming collagen microsponges in the openings of the PLGA knitted mesh. A Vicryl knitted mesh made of polyglactin 910 (a 90:10

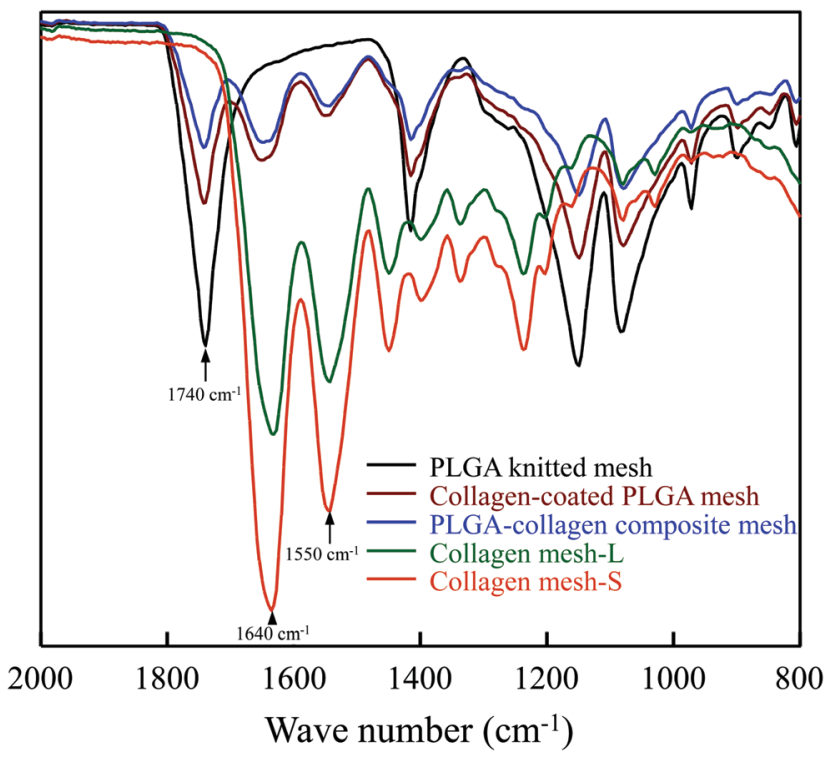

Fig. 2 ATR-FTIR spectra of PLGA mesh, collagen-coated PLGA mesh, $\mathrm{PLGA}-$ collagen composite mesh, collagen mesh- $\mathrm{L}$ and collagen mesh-S in the absorption band from 2000 to $800 \mathrm{~cm}^{-1}$.

copolymer of glycolic acid and lactic acid), as shown in Fig. 2a, was immersed in a porcine collagen acidic solution (type I, $\mathrm{pH}$ 3.0, $1.0 \mathrm{wt} \%$ ), frozen at $-80{ }^{\circ} \mathrm{C}$ for $12 \mathrm{~h}$ and freeze-dried under a vacuum of 0.2 Torr for $24 \mathrm{~h}$. Subsequently, the freeze-dried construct was placed in a closed box containing glutaraldehyde vapor saturated with a $25 \%$ glutaraldehyde aqueous solution at $37{ }^{\circ} \mathrm{C}$ for cross-linking for $4 \mathrm{~h}$. After cross-linking, the

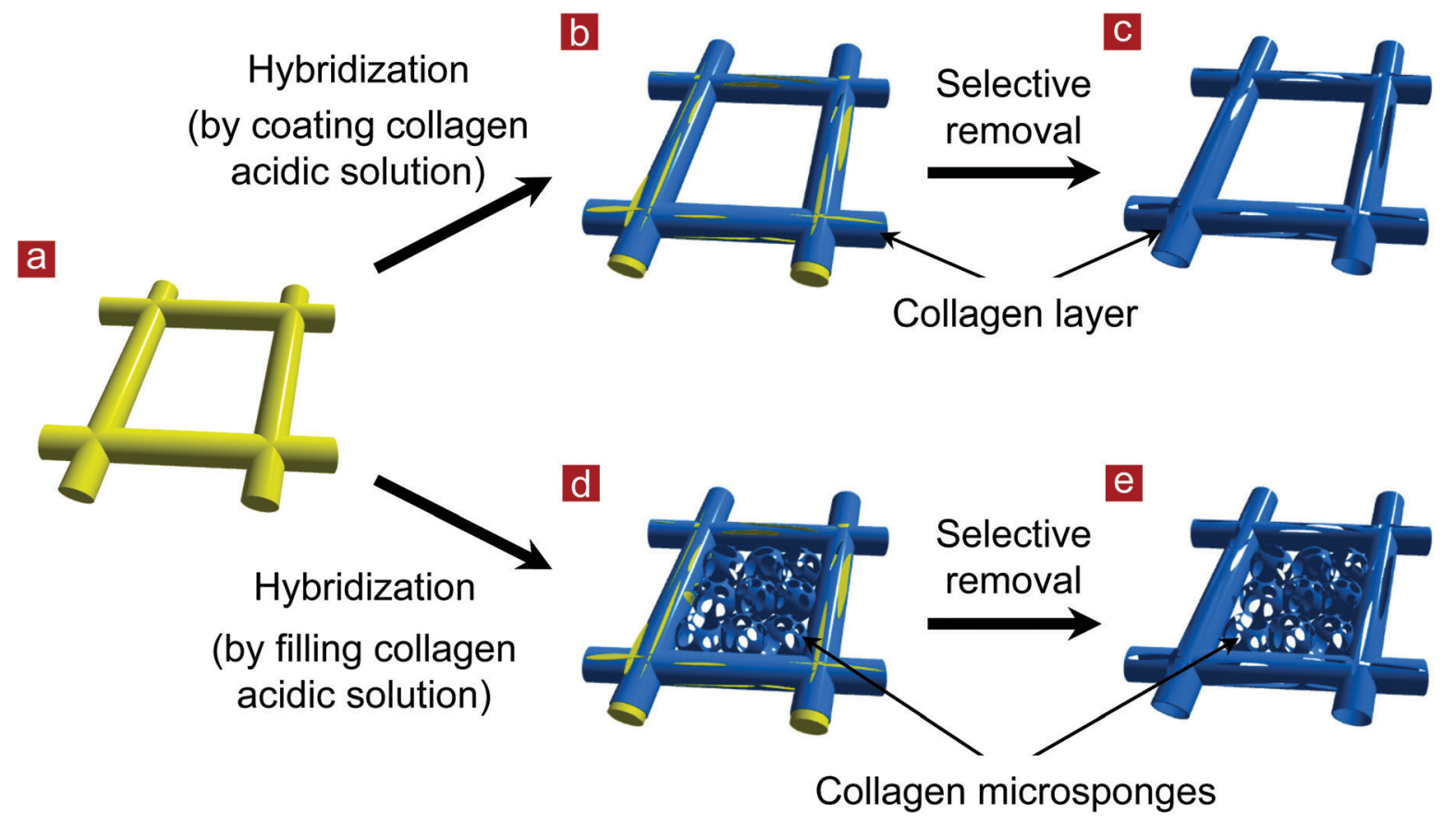

Fig. 1 Preparation scheme of mesh-type collagen scaffolds by a template leaching method. (a) PLGA mesh as a template. (b) Collagen-coated PLGA mesh. (c) Collagen mesh with large openings (collagen mesh-L). (d) PLGA-collagen composite mesh. (e) Collagen mesh with small openings (collagen mesh-S). 
PLGA-collagen composite mesh was treated with a $0.1 \mathrm{M}$ glycine aqueous solution to block unreacted aldehyde groups. Finally, the composite mesh was immersed in an aqueous solution of $0.5 \mathrm{M} \mathrm{Na}_{3} \mathrm{PO} 4$ and incubated at $37{ }^{\circ} \mathrm{C}$ for $48 \mathrm{~h}$ to selectively dissolve the PLGA mesh. ${ }^{22}$ Then, the mesh was washed with deionized water and freeze-dried.

Collagen mesh with a large opening (collagen mesh-L) was prepared by selectively removing PLGA from a collagen-coated PLGA mesh. Collagen was coated on the PLGA knitted mesh by embedding the PLGA mesh in a concentrated porcine collagen acidic solution (type I, pH 3.0, $5.0 \mathrm{wt} \%$ ) on a glass plate. The PLGA knitted mesh was then taken away from the glass plate and the excess collagen aqueous solution in the openings of the PLGA mesh was removed. A thick coating layer of collagen solution was formed on the PLGA fibers of the knitted mesh. The collagen-coated PLGA mesh was frozen at $-80{ }^{\circ} \mathrm{C}$ for $12 \mathrm{~h}$ and freeze-dried under a vacuum of 0.2 Torr for $24 \mathrm{~h}$. The subsequent treatments were the same as those of the PLGA-collagen composite mesh. Collagen mesh-L was obtained after selective removal of the PLGA mesh.

\section{Characterization of the meshes}

The structures of PLGA mesh, PLGA-collagen composite mesh, collagen-coated PLGA mesh, collagen mesh-S and collagen mesh-L were observed by a scanning electron microscope (SEM) operated at $10 \mathrm{kV}$. Small pieces of the meshes were attached to holders with carbon tape and coated with gold using a sputter coater.

Fourier transform infrared spectroscopy-attenuated total reflectance (FTIR-ATR) spectra of PLGA mesh, PLGA-collagen composite mesh, collagen-coated PLGA mesh, collagen mesh-S and collagen mesh-L were measured using an FT-IR spectrophotometer (IR Prestige-21, Shimadzu, Kyoto, Japan) equipped with a DuraSamplIR II diamond ATR accessory in the range of $2000-800 \mathrm{~cm}^{-1}$ at a resolution of $4 \mathrm{~cm}^{-1}$ and 32 scans.

\section{Cell culture}

Human foreskin fibroblasts were subcultured in 106S medium supplemented with $2(\mathrm{v} / \mathrm{v}) \%$ fetal bovine serum (FBS), $10 \mathrm{ng} \mathrm{mL}$ recombinant epidermal growth factor and $3 \mathrm{ng} \mathrm{mL}{ }^{-1}$ recombinant fibroblast growth factor-basic. Fibroblasts and medium were purchased from Cascade Biologics, Inc. (Portland, OR). The fibroblasts were subcultured once after reaching confluence. The subcultured fibroblasts were seeded in PLGA mesh, PLGA-collagen composite mesh, collagen-coated PLGA mesh, collagen mesh-S and collagen mesh-L. The meshes were cut into discs with a diameter of $1.2 \mathrm{~cm}$ and sterilized with ethylene oxide gas for cell culture. The meshes were placed in the wells of 24-well cell culture plates. Each of the meshes was covered with a glass ring with an internal diameter of $1.0 \mathrm{~cm}$ to protect against cell leakage during cell seeding. The subcultured fibroblasts were harvested by trypsin treatment and resuspended in serum-containing medium for cell seeding. The fibroblasts were seeded in each mesh by adding cell suspension solution to the glass rings that were placed on each mesh $\left(2.0 \times 10^{4}\right.$ cells per mesh). The cells were cultured in $106 \mathrm{~S}$ serumcontaining medium under a $5 \% \quad \mathrm{CO}_{2}$ atmosphere at $37{ }^{\circ} \mathrm{C}$.
The fibroblasts were seeded again on the opposite side of each mesh 3 hours after the first seeding except for the cell adhesion number and proliferation assay experiments. The glass rings were removed $6 \mathrm{~h}$ after cell seeding. The medium was changed every 2 days.

\section{Cell viability and proliferation}

The cell/mesh constructs after culture for 3.5 hours and 5 days were observed with an optical microscope. Cell viability was evaluated by live-dead staining using a Cellstain Double Staining Kit (Dojindo Laboratories, Tokyo, Japan). After the cells were cultured in the meshes for 7 days, the cell/mesh constructs were rinsed three times with buffer at $37{ }^{\circ} \mathrm{C}$ and incubated in serum-free medium containing calcein-AM $(2 \mu \mathrm{M})$ and PI $(4 \mu \mathrm{M})$ at $37{ }^{\circ} \mathrm{C}$ for $15 \mathrm{~min}$. The stained cells were observed with a fluorescence microscope.

Cell adhesion and proliferation were measured using the WST-1 assay (Roche Diagnostics, Indianapolis, IN, USA). After cell culture for 6 hours and 1, 2, 3, 4 and 5 days, the culture medium was aspirated, and $100 \mu \mathrm{L}$ of DMEM supplemented with $10 \%$ FBS was added along with $10 \mu \mathrm{L}$ of Cell Proliferation Reagent WST-1. For all time points, a standard curve was developed by plating $0.5 \times 10^{3}, 2.0 \times 10^{3}, 5.0 \times 10^{3}, 1.0 \times$ $10^{4}$ and $1.5 \times 10^{4}$ cells in $100 \mu \mathrm{L}$ of DMEM containing serum and $10 \mu \mathrm{L}$ of Cell Proliferation Reagent WST-1. The plates were then incubated for an additional $4 \mathrm{~h}$ at $37^{\circ} \mathrm{C}$. After incubation, the absorbance of the samples against the background control on a microtiter plate reader (Bio-Rad Benchmark Plus ${ }^{\mathrm{TM}}$ Microplate Spectrophotometer) was obtained at a wavelength of $440 \mathrm{~nm}$ with a reference wavelength of $650 \mathrm{~nm}$. The data after cell culture for 6 hours were used to calculate the cell adhesion number. Six samples under each condition were used for the measurement to calculate the means and standard deviations.

\section{Cell distribution and nuclear staining}

The cell distribution in the meshes was observed by SEM. After culture for 7 days, the cells cultured in the meshes were fixed with $0.25(\mathrm{v} / \mathrm{v}) \%$ glutaraldehyde solution in PBS at room temperature overnight. After being rinsed three times with PBS and once with deionized water, the cell/mesh constructs were freeze-dried. The morphology of the cells and meshes was observed using a scanning electron microscope after they were coated with Pt using a sputter coater.

For nuclear staining by DAPI, the cells cultured in the meshes for 7 days were fixed with phosphate-buffered $10 \%$ formalin at room temperature overnight. After being rinsed three times with PBS and once with deionized water, the cell/mesh constructs were stained using DAPI solution $\left(1.5 \mu \mathrm{L} \mathrm{mL}{ }^{-1}\right)$. The stained cells were observed using a fluorescence microscope.

\section{Histological staining}

After culture for 2 weeks, fibroblasts cultured in PLGA mesh, PLGA-collagen composite mesh and collagen mesh-S were fixed in PBS-buffered $10 \%$ formalin for histological staining. Then, the samples were dehydrated in ethanol, cleared in 
Lemosol and embedded in paraffin. They were sectioned at a thickness of $7 \mu \mathrm{m}$ and stained with hematoxylin and eosin.

\section{RNA isolation and real-time PCR}

For gene expression analysis, fibroblasts cultured in PLGA mesh, PLGA-collagen composite mesh, collagen mesh-S and TCPS for 1, 2, and 4 weeks were washed with PBS three times. The fibroblasts used for cell seeding were used as a control. The cells/mesh constructs were frozen in liquid nitrogen and then crushed by an electric crusher. The crushed samples were added to $1 \mathrm{~mL}$ of Isogen reagent (Nippon Gene, Toyama, Japan) to dissolve the RNA component. The cells cultured on TCPS and cells used for cell seeding were lysed in $1 \mathrm{~mL}$ of Isogen reagent. Total RNA was extracted following the manufacturer's protocol. One microgram of total RNA was reverse transcribed into cDNA using random hexamers (Applied Biosystems) in a $20 \mu \mathrm{L}$ reaction. An aliquot $(1 \mu \mathrm{L})$ of 10 -fold-diluted reaction solution was used for each $25 \mu \mathrm{L}$ real-time PCR together with $300 \mathrm{nM}$ forward and reverse primers, $150 \mathrm{nM}$ probe and qPCR Master Mix (EUROGENETIC). TaqMan ${ }^{\circledR}$ probes and primer pairs for collagen type I (COL1A2, forward: 5'-CAGCCGCTTCACCTACACGC-3', reverse: $5^{\prime}$ TTTTGTATTCAATCACTGTCTTGCC-3', probe: $5^{\prime}$-CCGGTGTGACTCGTGCAGCCATC-3'), ${ }^{23}$ collagen type IV (COLAA2, assay identification number Hs01098873_m1), laminin (LAMA1, Hs00300550_m1), vascular endothelial growth factor (VEGFA, Hs00900054_m1) and fibroblast growth factor-7 (FGF7, Hs00384281_m1), which are assay-on-demand gene expression products, were obtained from Applied Biosystems and used according to the manufacturer's instructions. Real-time PCR analysis was performed using the 7500 Real-Time PCR System (Applied Biosystems). After an initial incubation step of $2 \mathrm{~min}$ at $50{ }^{\circ} \mathrm{C}$ and denaturation for $10 \mathrm{~min}$ at $95{ }^{\circ} \mathrm{C}, 40$ cycles $\left(95^{\circ} \mathrm{C}\right.$ for $15 \mathrm{~s}, 60{ }^{\circ} \mathrm{C}$ for $1 \mathrm{~min}$ ) of PCR were performed, and reactions were performed in triplicate. $18 \mathrm{~S}$ recombinant RNA levels were used as endogenous controls, and gene expression levels relative to GAPDH were calculated using a comparative Ct method. Three samples under each condition were used for measurements to calculate the means and standard deviations.

\section{Statistical analysis}

All quantitative analysis experiments were performed in triplicate. One-way analysis of variance (ANOVA) was used to calculate the significance of all the data using KyPlot 6.0 (KyensLab Inc., Tokyo, Japan). The results are reported as the mean \pm standard deviation (SD). Significant differences are shown as ${ }^{*}(p<0.05),{ }^{* *}(p<$ $0.01)$ and ${ }^{* *}, p<0.001$.

\section{Results}

\section{Characterization of collagen meshes}

Two types of collagen meshes were prepared using PLGA knitted mesh as a template (Fig. 1). One type was collagen mesh with large openings (collagen mesh-L), which was prepared by selectively dissolving the PLGA mesh template from a collagen-coated PLGA mesh. The other type was collagen mesh with small openings (collagen mesh-S), which was prepared by selectively dissolving the PLGA template from a PLGA-collagen composite mesh. The collagen-coated PLGA mesh was prepared by coating collagen on the skeleton of the PLGA mesh template. The PLGA-collagen composite mesh was fabricated by forming collagen microsponges in the openings of the PLGA mesh.

To confirm whether the PLGA template was completely removed from the collagen mesh-L and collagen mesh-S, ATR-FTIR spectra were measured before and after removal of the PLGA template (Fig. 2). The typical peak at $1740 \mathrm{~cm}^{-1}$ assigned to the $\mathrm{C}=\mathrm{O}$ stretching vibration of ester bonds in PLGA was detected in the PLGA, collagen-coated PLGA and PLGA-collagen composite meshes. The two representative peaks at $1640 \mathrm{~cm}^{-1}$ and $1550 \mathrm{~cm}^{-1}$ that are assigned to amides I and II of collagen, respectively, were detected in the collagencoated PLGA mesh, PLGA-collagen composite mesh, collagen mesh-L and collagen mesh-S. Disappearance of the ester carbonyl stretch peak in the collagen mesh-L and collagen mesh-S spectra indicated the complete removal of the PLGA template. The alkaline aqueous solution could dissolve the synthetic PLGA but had no evident effect on collagen, resulting in selective dissolution of PLGA from the collagen-coated PLGA and PLGA-collagen composite meshes.

SEM observation showed that the collagen-coated PLGA mesh had the same morphology as that of the PLGA knitted mesh. Collagen was coated on the PLGA fibers of the knitted mesh (Fig. 3a and b). After removal of the PLGA mesh template, collagen mesh-L had the same large openings as collagencoated PLGA mesh (Fig. 3c). The collagen coated on the PLGA fibers remained to form the collagen mesh-L structure. Observation of the cross-section $\left(60^{\circ}\right.$ view) of the meshes showed that removal of the PLGA mesh template left the cavity, resulting in the formation of a microtubular structure of collagen (Fig. 3d and e). The cavity in microtubular collagen was a negative replica of the PLGA mesh template. Collagen mesh-L was composed of microtubular collagen.

Collagen mesh-S also showed the same morphology as the PLGA-collagen composite mesh (Fig. 4). After removal of the PLGA mesh template, the microtubular collagen formed the same mesh structure as that of the PLGA mesh. Furthermore, web-like collagen microsponges remained in the openings of

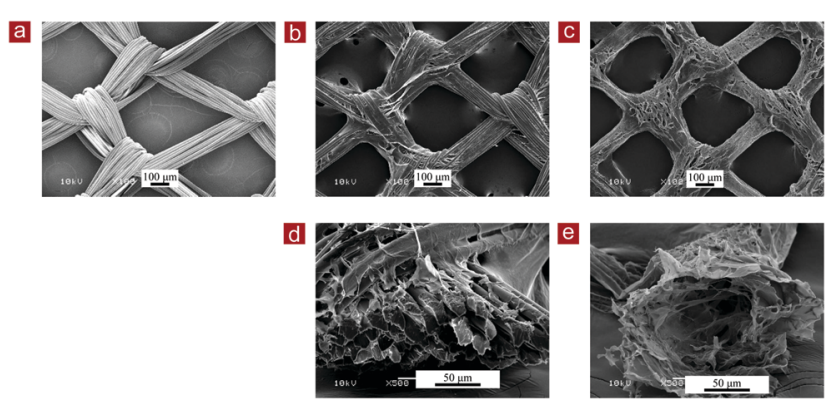

Fig. 3 SEM images of the top surface view $(a-c)$ and the cross-sectional view $\left(60^{\circ}\right.$ view) ( $d$ and e) of the PLGA mesh (a), collagen-coated PLGA mesh ( $b$ and $d$ ) and collagen mesh- $L$ (c and $e$ ). 

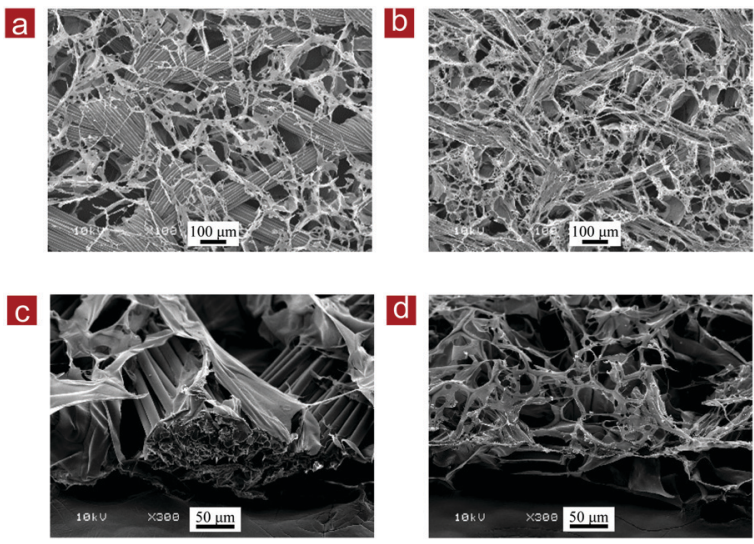

Fig. 4 SEM images of the top surface view ( $a$ and $b$ ) and the crosssectional view $\left(60^{\circ}\right.$ view) (c and d) of the PLGA-collagen composite mesh $(a$ and $c$ ) and collagen mesh-S ( $b$ and $d)$.

the microtubular collagen mesh (Fig. $4 \mathrm{~b}$ and d). Therefore, collagen mesh-S had a hierarchical structure that was composed of collagen mesh-L and collagen microsponges in the openings of collagen mesh-L. Collagen mesh-S had smaller openings and more surface area for cell adhesion and matrix deposition than collagen mesh-L. The thickness of collagen mesh-L and collagen mesh-S was about $150 \mu \mathrm{m}$.

\section{Cell culture in collagen meshes}

Collagen mesh-L and collagen mesh-S were used for the culture of human skin fibroblasts and were compared with PLGA mesh, collagen-coated PLGA mesh, PLGA-collagen composite mesh and polystyrene cell culture plates. Observation with an optical microscope indicated that the fibroblasts immediately after cell seeding were captured by the web-like collagen microsponges in the collagen mesh-S and PLGA-collagen composite mesh but fell through the openings of the collagen mesh-L, PLGA mesh and collagen-coated PLGA mesh (Fig. 5). The fibroblasts in the collagen mesh-L, PLGA mesh and collagen-coated PLGA mesh grew along the polymer fibers toward the centers of the mesh openings. The cells in the collagen mesh-S and PLGA-collagen composite mesh grew homogenously.
The number of fibroblasts that adhered to these scaffolds after 6 hours was measured. The initially adhered cell number in the scaffolds was in a decreasing order of PLGA-collagen composite mesh, collagen mesh-S, collagen-coated PLGA mesh, collagen mesh-L and PLGA mesh (Fig. 6a). Cell adhesion on the TCPS was the highest. Collagen has a cell adhesion moiety, such as an arginine-glycine-aspartic acid (RGD) tripeptide; thus, collagen-containing scaffolds promote cell adhesion through the specific interaction of cells with the RGD tripeptide. The collagen mesh-S and PLGA-collagen composite mesh had more surfaces for cell adhesion than collagen mesh-L, collagen-coated PLGA mesh and PLGA mesh.

Cell proliferation was evaluated by WST-1 assay (Fig. 6b). The cells cultured on the TCPS showed the highest proliferation. The cells cultured in the collagen mesh-S and PLGA-collagen composite mesh showed the same level proliferation, which was second highest after TCPS. Cell proliferation levels in the collagen mesh-L and collagen-coated PLGA mesh were the third highest, and cell proliferation in the PLGA mesh was the lowest. The collagen mesh-S and PLGA-collagen composite mesh had more promotive effects on cell adhesion and proliferation than the collagen mesh-L PLGA mesh and collagen-coated PLGA mesh.

SEM observation of the cells/mesh constructs after 7 days of culture showed that the fibroblasts proliferated and occupied the spaces in the meshes (Fig. 7a-e). The cells occupied almost all the spaces in the collagen mesh-S and PLGA-collagen composite mesh, while large spaces remained in the PLGA mesh and collagen-coated PLGA mesh. The space-occupying speed of the cultured fibroblasts was faster in the collagen mesh-S and PLGA-collagen composite mesh than in the other meshes. The results indicated that the web-like collagen microsponges in the collagen mesh-S and PLGA-collagen composite mesh facilitated homogenous cell distribution and extracellular matrix deposition.

The distribution of fibroblasts in the scaffolds was further confirmed by nuclear staining. Staining with DAPI staining showed that the fibroblasts in the collagen mesh-L, PLGA mesh and collagen-coated PLGA mesh were predominantly distributed surrounding the mesh fibers, while the cells in the collagen mesh-S and PLGA-collagen composite mesh were
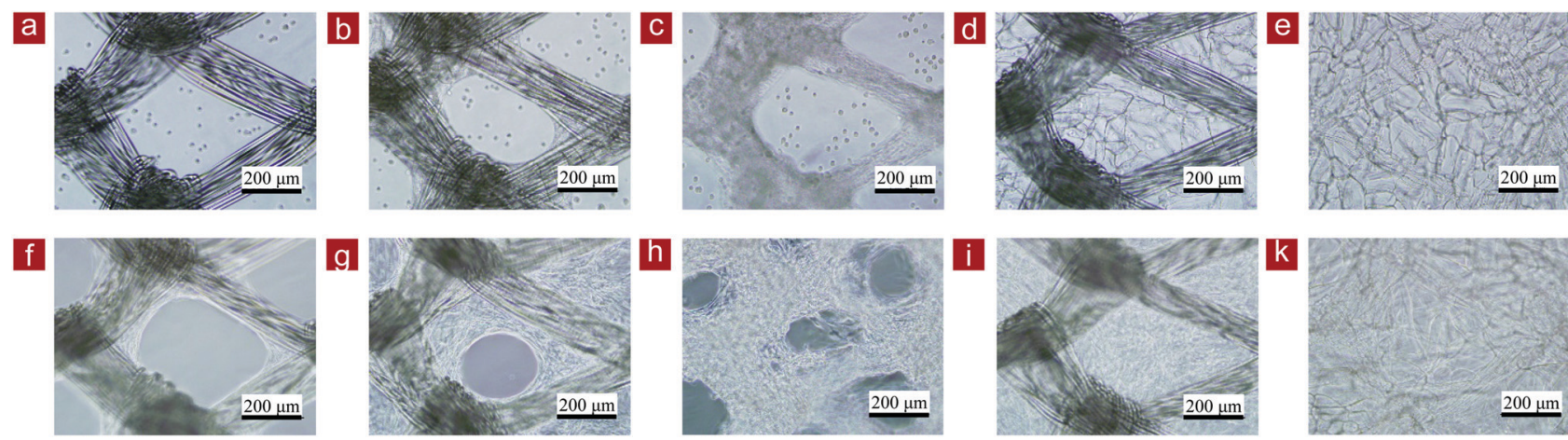

Fig. 5 Phase-contrast photomicrographs of fibroblasts cultured in PLGA mesh (a and f), collagen-coated PLGA mesh (b and g), collagen mesh-L (c and h), PLGA-collagen composite mesh ( $d$ and i) and collagen mesh-S (e and k) for 3.5 hours (a-e) and 5 days ( $f-k$ ). 

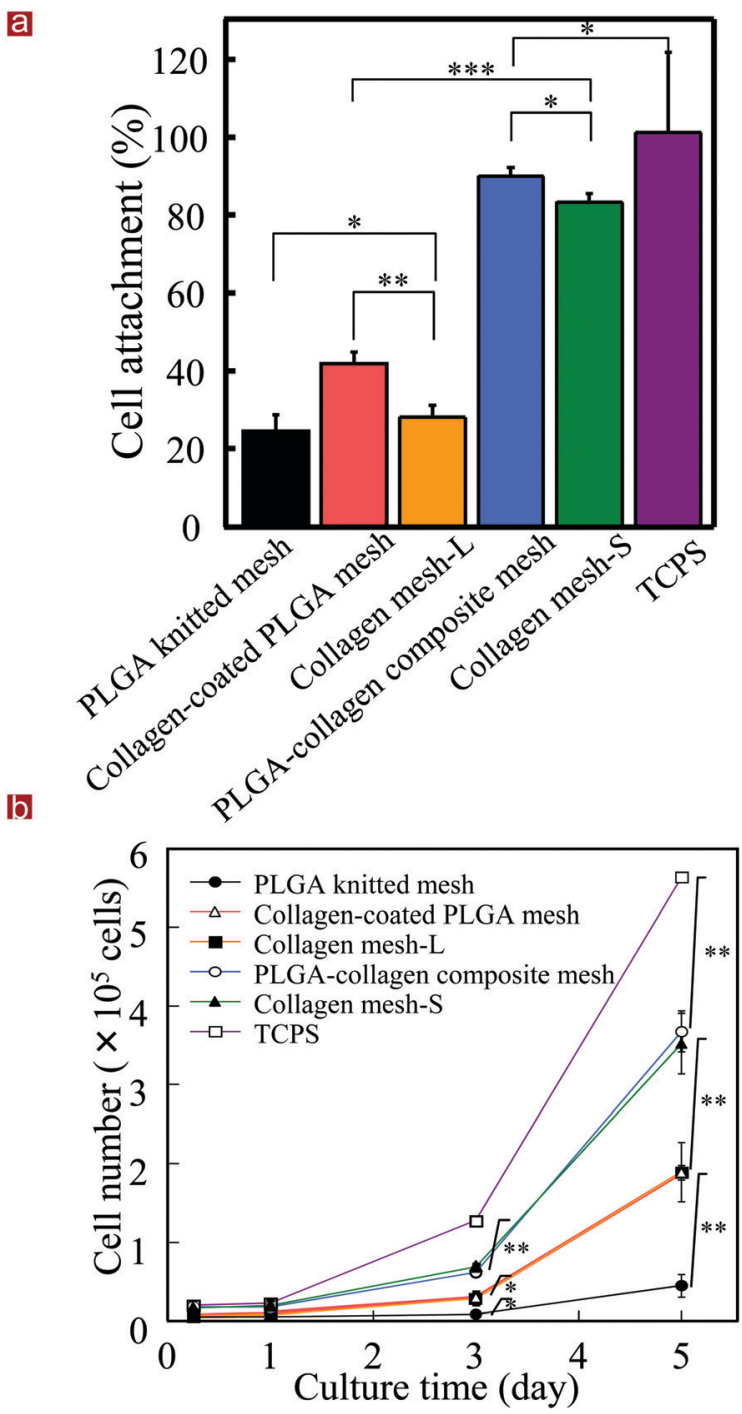

Fig. 6 Adhesion of fibroblasts in the scaffolds after culture for 6 hours (a) and proliferation of fibroblasts cultured in the scaffolds for 5 days (b). TCPS was used as a control. Data represent the mean \pm SD, $n=6$. Significant difference: ${ }^{*}, p<0.05 ;{ }^{* *}, p<0.01 ;{ }^{* *}, p<0.001$

distributed homogenously throughout the scaffolds (Fig. $7 \mathrm{f}-\mathrm{j}$ ). The viability of cells in the scaffolds was further confirmed by live/dead staining. The cells were alive (green), with almost no dead cells (red) detected in these scaffolds, indicating high cell viability in the scaffolds (Fig. 7k-o).

\section{Regeneration of dermal tissue and gene expression}

Collagen mesh-S was used for the long-term culture of fibroblasts for in vitro dermal tissue regeneration because it showed the highest effects on cell adhesion and proliferation. After 2 weeks of culture, the cells/mesh constructs were sectioned and examined by HE staining (Fig. 8). The results showed that the dermal tissue formed in the PLGA mesh was thick near the PLGA mesh fibers and became thin in the centers of the openings. In contrast, the thickness of the dermal tissues formed in the collagen-S and PLGA-collagen composite mesh was relatively uniform. The PLGA and collagen fibers of the scaffolds could be observed in the stained images, which indicated the scaffolds were not degraded after 2 weeks culture. Large voids were observed in the dermal tissues regenerated in the PLGA mesh and PLGA-collagen composite mesh, which was due to the existence of PLGA fibers in the scaffolds. Conversely, the dermal tissue regenerated in collagen mesh-S was more homogenous without large voids. The results indicated that collagen mesh-S facilitated cell adhesion, promoted cell proliferation and was beneficial for homogenous dermal tissue regeneration.

The gene expression levels of type I collagen, type IV collagen, laminin, FGF-7 and VEGF were examined by realtime PCR after fibroblasts were cultured in collagen mesh-S, PLGA mesh and PLGA-collagen composite mesh for 1, 2 and 4 weeks (Fig. 9). TCPS was used as a control. Fibroblasts cultured in collagen mesh-S showed the highest expression levels of these genes. The expression levels of these genes in the collagen mesh-S were significantly higher than those in the PLGA mesh, PLGA-collagen composite mesh and TCPS. Moreover, the expression levels of these genes in collagen mesh-S increased with time during the 4 weeks of culture. The results indicated that collagen mesh-S promoted the expression of dermal tissuerelated genes and promoted dermal tissue regeneration.

\section{Discussion}

Scaffolds for tissue engineering should not only support smooth cell seeding and cell distribution but also promote cell proliferation and matrix secretion. It is important to precisely control the porous architecture of scaffolds to elicit cell-cell interactions and cell-ECM interactions to guide new tissue regeneration. In this study, we demonstrated a template leaching method for the preparation of mesh-type collagen scaffolds. Mesh-type scaffolds have been broadly used in tissue engineering applications. However, it has been difficult to prepare thin collagen meshes. Using PLGA mesh as a template, collagen mesh-L and collagen mesh-S were prepared. The PLGA mesh with a continuous design structure was used as a selectively removable template. The structures of collagen mesh-L and collagen mesh-S could be controlled by the structure of the PLGA mesh template. Collagen mesh-L had a mesh-like structure of microtubular collagen (Fig. 3). Collagen microsponges were further formed in the openings of the microtubular collagen network to construct the collagen mesh-S (Fig. 4). Collagen mesh-S was composed of collagen mesh-L and collagen microsponges. Therefore, collagen mesh-S had smaller pores and more surfaces than collagen mesh-L.

Collagen meshes were used for the culture of fibroblasts for their possible application in dermal tissue regeneration. Both collagen mesh-L and collagen mesh-S promoted cell adhesion and proliferation at the same levels of their respective precursor scaffolds, collagen-coated PLGA mesh and PLGAcollagen composite mesh (Fig. 5 and 6). The effects were much more evident than that of PLGA mesh. The RGD sequence in the collagen should contribute to the promotive effects because 

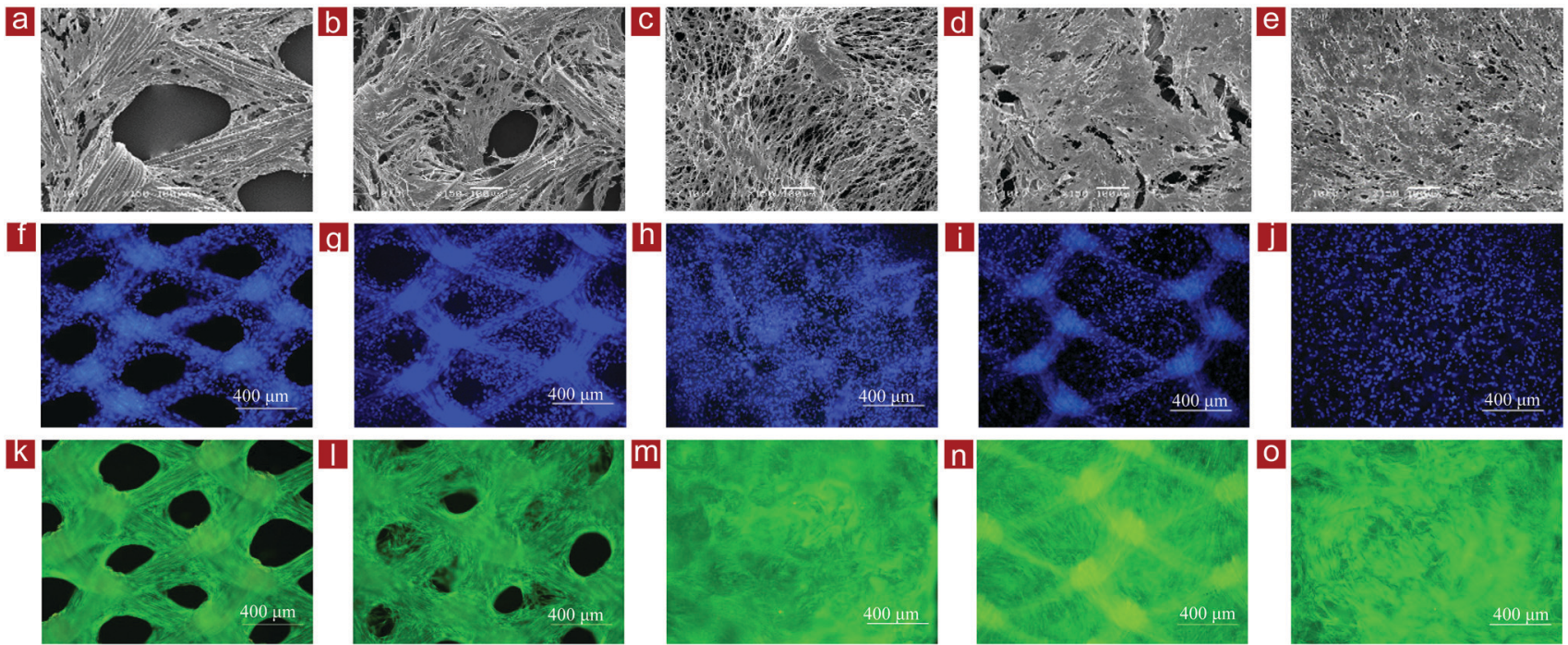

Fig. 7 SEM images $(a-e)$, nuclear staining $(f-j)$ and live/dead staining $(k-0)$ of fibroblasts cultured in PLGA mesh $(a, f, k)$, collagen-coated PLGA mesh $(b$, $\mathrm{g}, \mathrm{l})$, collagen mesh-L $(c, h, m)$, PLGA-collagen composite mesh $(\mathrm{d}, \mathrm{i}, \mathrm{n})$, and collagen mesh-S $(\mathrm{e}, \mathrm{j}, \mathrm{o})$ for 7 days. Nuclei are stained blue. Living cells are stained green, while dead cells are stained red.

a

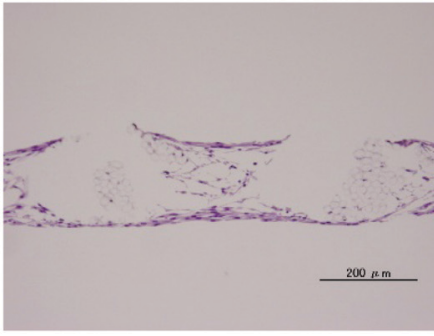

d

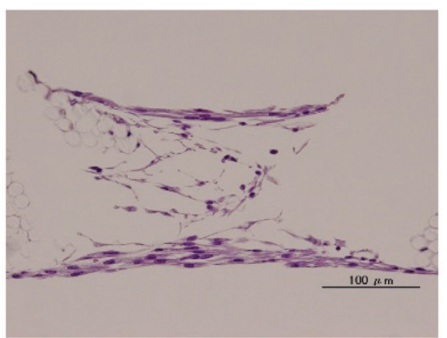

b

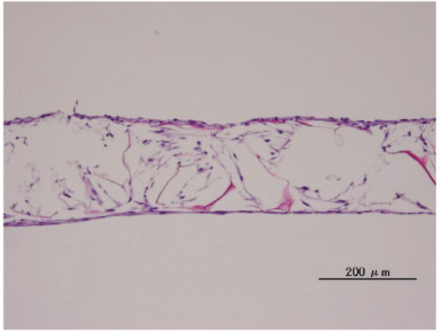

e

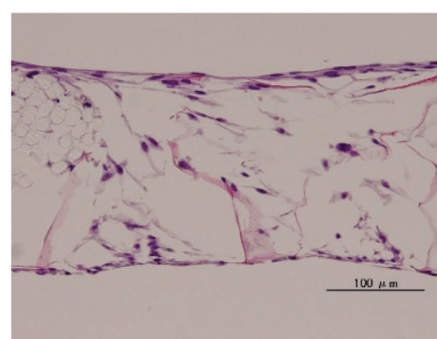

C

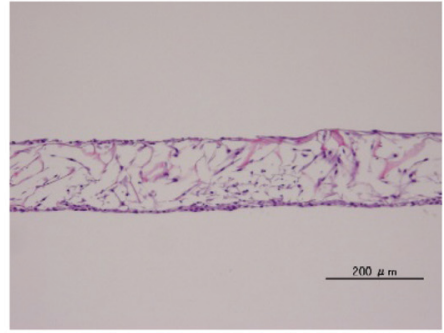

f

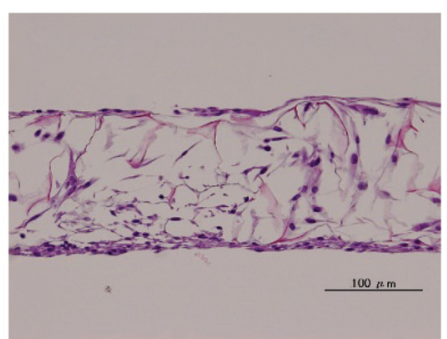

Fig. 8 Hematoxylin/eosin staining of cells/mesh constructs after culture in PLGA mesh (a and d), PLGA-collagen composite mesh (b and e) and collagen mesh-S ( $c$ and f) for 2 weeks. The original magnifications are $\times 100(a-c)$ and $\times 200(d-e)$.

the collagen mesh-L, collagen mesh-S, collagen-coated PLGA mesh and PLGA-collagen composite mesh all contained collagen. It has been reported that the RGD sequence is beneficial for cell adhesion and proliferation. ${ }^{24,25}$ Compared to collagen mesh-L and collagen-coated PLGA mesh, collagen mesh-S and PLGA-collagen composite mesh promoted cell adhesion and proliferation more strongly. This is because collagen microsponges are formed in the openings of the microtubular collagen network or the PLGA mesh.

The fibroblasts in the collagen mesh-S and PLGA-collage composite mesh became layered structures more quickly than those in the collagen mesh-L, collagen-coated PLGA mesh and PLGA mesh (Fig. 7 and 8). The collagen mesh-S and PLGAcollage composite mesh had smaller pores and more surface areas for cell adhesion and proliferation. However, most of the fibroblasts passed through the openings of the collagen mesh-L, collagen-coated PLGA mesh and PLGA mesh that had large openings and therefore failed to attach to the scaffolds (Fig. 5). The cells grew along the mesh skeleton toward the centers of the mesh openings. In contrast, with the collagen mesh-S and PLGA-collagen composite mesh, the fibroblasts were caught by web-like collagen microsponges. The fibroblasts in the collagen mesh-S and PLGA-collagen composite mesh adhered and distributed homogeneously and therefore grew 

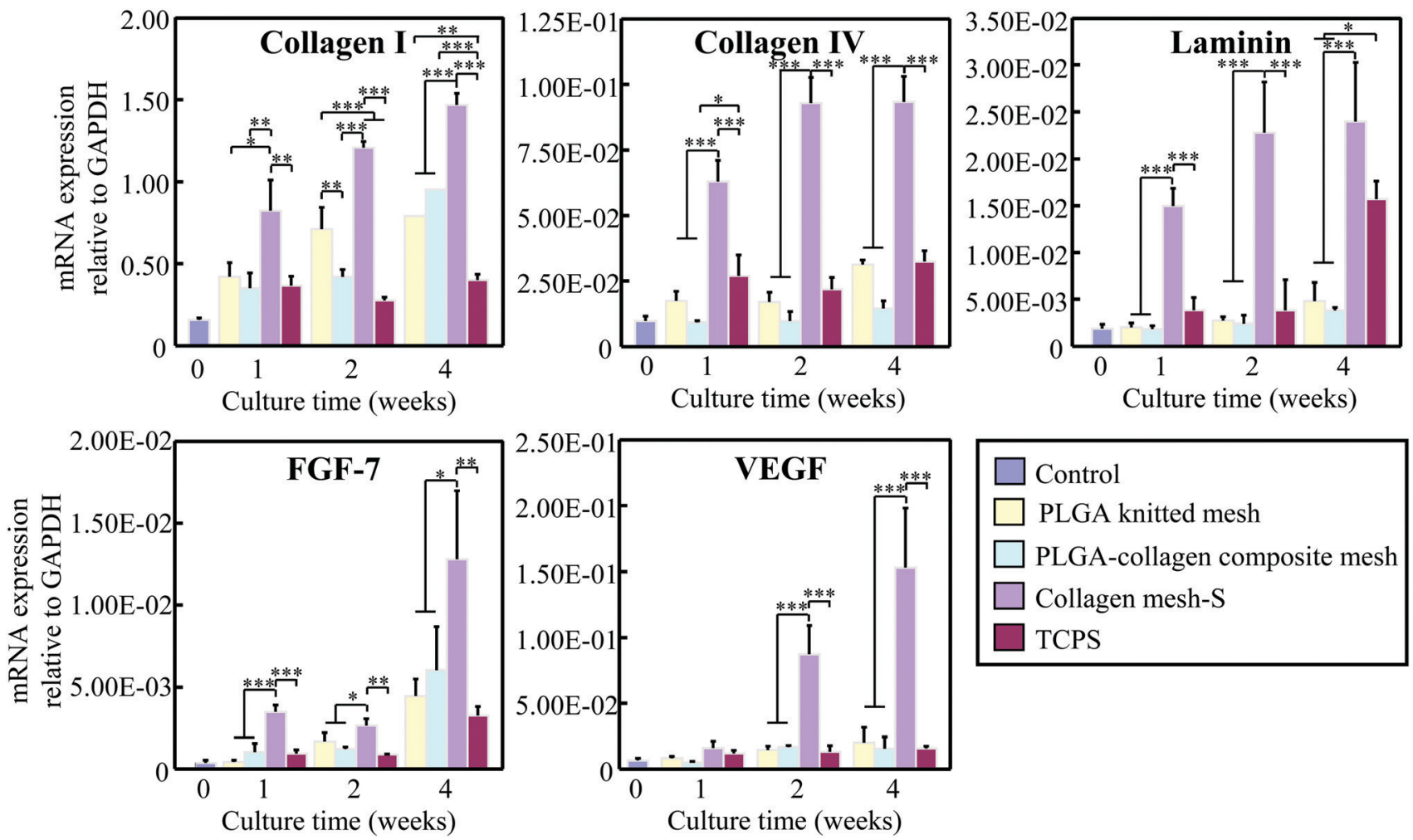

Fig. 9 Expression levels of genes encoding type I collagen, type IV collagen, laminin, FGF-7 and VEGF after fibroblasts were cultured in PLGA mesh, PLGA-collagen composite mesh and collagen mesh-S for 1, 2 and 4 weeks. Fibroblasts used for cell seeding and TCPS was used as a control. Data were normalized to the expression of GAPDH. Data represent the mean $\pm \mathrm{SD}, n=3$. Significant difference: ${ }^{*}, p<0.05 ;{ }^{* *}, p<0.01 ;{ }^{* *}, p<0.001$.

homogeneously to generate evenly layered structures in the scaffolds.

The expression levels of type I collagen, type IV collagen, laminin, FGF-7 and VEGF by fibroblasts cultured in collagen mesh-S were significantly higher than those in fibroblasts cultured in PLGA-collagen composite mesh and PLGA mesh. Type I collagen is the dominant component of dermal tissue. ${ }^{26}$ Laminin and type IV collagen are the two primary basement membrane proteins of skin. ${ }^{27}$ Higher expression levels of these genes could promote the proliferation of fibroblasts and the regeneration of dermal tissue. Furthermore, FGF-7 and VEGF are important factors for angiogenesis and re-epithelialization. ${ }^{28}$ High gene expression levels of FGF-7 and VEGF indicated that collagen mesh-S could induce more rapid vascularization and re-epithelization.

The positive effects of collagen mesh-S should be due to the synergistic effects of the microtubular collagen network and collagen microsponges formed in the openings of the microtubular collagen network. The microtubular collagen network could avoid the negative influence of PLGA. Degradation of PLGA in the scaffolds could result in large voids in the regenerated dermal tissue (Fig. 8). Degradation of PLGA has been reported to result in low local $\mathrm{pH}$, which may have a negative effect on cell functions. ${ }^{29}$ Collagen mesh-S did not have such PLGA-derived negative influences. Meanwhile, the collagen microsponges in the openings of the microtubular collagen network provided additional small pores to entrap the cells and more surfaces for cell adhesion. The homogeneous distribution of cells and extracellular matrices in collagen mesh-S could benefit cell-cell interactions and cell-ECM interactions, therefore promoting high expression of related genes and matrix secretion. The collagen mesh-S could facilitate dermal tissue regeneration. Compared to the PLGA knitted mesh and PLGA-collagen composite mesh, the collagen meshes were very soft. They were mechanically too weak for application in load-bearing tissue regeneration. However, the collagen meshes such as collagen mesh-S could be used for regeneration of non-load-bearing tissues such as dermal tissue, cornea and capillaries.

\section{Conclusions}

Collagen meshes with large and small openings were prepared using PLGA as a template. The porous structures of collagen meshes could be controlled by their precursor meshes. Collagen mesh-S had a hierarchical structure that was composed of collagen mesh-L and collagen microsponges formed in the openings of collagen mesh-L. Both collagen mesh-L and collagen mesh-S could support fibroblast adhesion and proliferation. The promotive effect of collagen mesh-S was stronger than that of collagen mesh-L. Collagen mesh-S facilitated even cell distribution and homogenous dermal tissue regeneration. Collagen mesh-S should be useful for 
dermal tissue regeneration, and the template method is useful for the preparation of mesh-like collagen scaffolds for tissue engineering applications.

\section{Conflicts of interest}

There are no conflicts to declare.

\section{Acknowledgements}

This research was supported by JSPS KAKENHI Grant Number 19H04475 and 21H03830.

\section{Notes and references}

1 B. Orel, M. Giovine and M. Ilan, Mar. Drugs, 2021, 19.

2 E. Rezvani Ghomi, N. Nourbakhsh, M. Akbari Kenari, M. Zare and S. Ramakrishna, J. Biomed. Mater. Res., Part B, 2021, 109, 1986-1999.

3 M. R. El-Aassar, O. M. Ibrahim and Z. H. Al-Oanzi, Polymers, 2021, 13.

4 J. Yan, C. Liu, C. Tu, R. Zhang, X. Tang, H. Li, H. Wang, Y. Ma, Y. Zhang and H. Wu, Stem Cell Res. Ther., 2021, 12, 1-21.

5 Y. Xie, K. Lee, X. Wang, T. Yoshitomi, N. Kawazoe, Y. Yang and G. Chen, J. Mater. Chem. B, 2021, 9, 8491-8500.

6 V. Irawan, T.-C. Sung, A. Higuchi and T. Ikoma, Tissue Eng. Regener. Med., 2018, 15, 673-697.

7 Z. Li, T. Du, C. Ruan and X. Niu, Bioact. Mater., 2021, 6, 1491-1511.

8 Y. Chen, N. Kawazoe and G. Chen, Acta Biomater., 2018, 67, 341-353.

9 A. Wubneh, E. K. Tsekoura, C. Ayranci and H. Uludağ, Acta Biomater., 2018, 80, 1-30.

10 Y. Chen, K. Lee, N. Kawazoe, Y. Yang and G. Chen, J. Mater. Chem. B, 2019, 7, 7195-7206.

11 P. Bajaj, R. M. Schweller, A. Khademhosseini, J. L. West and R. Bashir, Annu. Rev. Biomed. Eng., 2014, 16, 247-276.

12 A. Dewle, N. Pathak, P. Rakshasmare and A. Srivastava, ACS Biomater. Sci. Eng., 2020, 6, 779-797.
13 Z. Rao, T. Lin, S. Qiu, J. Zhou, S. Liu, S. Chen, T. Wang, X. Liu, Q. Zhu and Y. Bai, Mater. Sci. Eng., C, 2021, 120, 111791.

14 H. Zhang, I. Hussain, M. Brust, M. F. Butler, S. P. Rannard and A. I. Cooper, Nat. Mater., 2005, 4, 787-793.

15 K. Qin, C. Parisi and F. M. Fernandes, J. Mater. Chem. B, 2021, 9, 889-907.

16 Y. He, M. Tian, X. Li, J. Hou, S. Chen, G. Yang, X. Liu and S. Zhou, Adv. Healthcare Mater., 2021, 2102236.

17 D. Huang, T. Liu, J. Liao, S. Maharjan, X. Xie, M. Pérez, I. Anaya, S. Wang, A. T. Mayer and Z. Kang, Proc. Natl. Acad. Sci. U. S. A., 2021, 118.

18 Q. Zhang, H. Lu, N. Kawazoe and G. Chen, Acta Biomater., 2014, 10, 2005-2013.

19 Y. S. Zhang, C. Zhu and Y. Xia, Adv. Mater., 2017, 29, 1701115.

20 M. J. Dewey, D. J. Milner, D. Weisgerber, C. L. Flanagan, M. Rubessa, S. Lotti, K. M. Polkoff, S. Crotts, S. J. Hollister, M. B. Wheeler and B. A. C. Harley, Biofabrication, 2021, 14, 014102.

21 E. McKenna, T. J. Klein, M. R. Doran and K. Futrega, Biofabrication, 2019, 12, 015015.

22 H. Lu, T. Hoshiba, N. Kawazoe, I. Koda, M. Song and G. Chen, Biomaterials, 2011, 32, 9658-9666.

23 T. Hoshiba, H. Lu, T. Yamada, N. Kawazoe, T. Tateishi and G. Chen, Biotechnol. Prog., 2011, 27, 788-795.

24 E. García-Gareta, A. Levin and L. Hook, Biotechnol. Bioeng., 2019, 116, 1102-1115.

25 E. Y. Jeon, B.-H. Choi, D. Jung, B. H. Hwang and H. J. Cha, Biomaterials, 2017, 134, 154-165.

26 A. El Ghalbzouri, S. Commandeur, M. H. Rietveld, A. A. Mulder and R. Willemze, Biomaterials, 2009, 30 71-78.

27 G. Erdag, D. A. Medalie, H. Rakhorst, G. G. Krueger and J. R. Morgan, Mol. Ther., 2004, 10, 76-85.

28 Y. Bayir, H. Un, R. A. Ugan, E. Akpinar, E. Cadirci, I. Calik and Z. Halici, Burns, 2019, 45, 1410-1417.

29 K.-S. Park, B.-J. Kim, E. Lih, W. Park, S. H. Lee, Y. K. Joung and D. K. Han, Acta Biomater., 2018, 73, 204-216. 